University of Nebraska - Lincoln

DigitalCommons@University of Nebraska - Lincoln

2019

\title{
Molecular Symmetry-Mixed Dichroism in Double Photoionization of $\mathrm{H}_{2}$
}

Jean Marcel Ngoko Djiokap

Alexei V. Meremianin

N. L. Manakov

L. B. Madsen

S. X. Hu

See next page for additional authors

Follow this and additional works at: https://digitalcommons.unl.edu/physicsstarace

Part of the Atomic, Molecular and Optical Physics Commons, Elementary Particles and Fields and String Theory Commons, and the Plasma and Beam Physics Commons

This Article is brought to you for free and open access by the Research Papers in Physics and Astronomy at DigitalCommons@University of Nebraska - Lincoln. It has been accepted for inclusion in Anthony F. Starace Publications by an authorized administrator of DigitalCommons@University of Nebraska - Lincoln. 
Authors

Jean Marcel Ngoko Djiokap, Alexei V. Meremianin, N. L. Manakov, L. B. Madsen, S. X. Hu, and Anthony F. Starace 


\title{
Molecular Symmetry-Mixed Dichroism in Double Photoionization of $\mathbf{H}_{2}$
}

\author{
J. M. Ngoko Djiokap $\odot,{ }^{1, *}$ A. V. Meremianin, ${ }^{2}$ N. L. Manakov, ${ }^{2}$ L. B. Madsen, ${ }^{3}$ S. X. Hu, ${ }^{4}$ and Anthony F. Starace $\odot^{1,}$ \\ ${ }^{1}$ Department of Physics and Astronomy, University of Nebraska, Lincoln, Nebraska 68588-0299, USA \\ ${ }^{2}$ Department of Physics, Voronezh State University, Voronezh 394006, Russia \\ ${ }^{3}$ Department of Physics and Astronomy, Aarhus University, DK-8000 Aarhus C, Denmark \\ ${ }^{4}$ Laboratory for Laser Energetics, University of Rochester, Rochester, New York 14623-1299, USA
}

(Received 24 May 2019; revised manuscript received 24 June 2019; published 2 October 2019)

\begin{abstract}
Dichroism in double photoionization of $\mathrm{H}_{2}$ molecules by elliptically polarized extreme ultraviolet pulses is formulated analytically as a sum of atomiclike dichroism (AD) and molecular symmetry-mixed dichroism (MSMD) terms. The MSMD originates from an interplay of ${ }^{1} \Sigma_{u}^{+}$and ${ }^{1} \Pi_{u}^{+}$continuum molecular ionization amplitudes. For detection geometries in which the AD vanishes, numerical results for the sixfold differential probabilities for opposite pulse helicities show that the MSMD is significant in the electron momentum and angular distributions and is controllable by the ellipticity.
\end{abstract}

DOI: 10.1103/PhysRevLett.123.143202

Single-photon double photoionization (DPI) of an atom is a fundamental process in which electron correlation plays a crucial role [1,2]. For circularly polarized photons, a circular dichroism (CD) effect was predicted for this process [3]: the two-electron angular distributions for rightand left-handed circular polarization differ. In general, dichroic phenomena in photoionization occur for both achiral targets [3-13] and chiral targets [14-16]. A key difference is that dichroic effects for achiral targets exist in the angular distributions, but vanish in the total cross sections, whereas for chiral targets they exist in both [12]. Applications of CD effects extend from studying the structures of proteins (e.g., [17]) to controlling dynamics in chiral molecules (e.g., [18]) or in magnetic materials (e.g., $[19,20])$.

Although CD in the DPI angular distributions of $\mathrm{He}$ atoms is well studied both theoretically $[3-5,12,13]$ and experimentally [6-11], there are very few studies of CD effects in DPI of the fundamental two-electron molecule, $\mathrm{H}_{2}$. Expressions for CD in DPI of $\mathrm{H}_{2}$ have been formulated $[21,22]$, but no predictions exist for its magnitude. Measurements of $\mathrm{H}_{2}$ DPI angular distributions have focused either on comparisons with DPI of $\mathrm{He}$ [23,24] or on two-center interference effects in angular distributions produced by high-energy, circularly polarized photons for the case of extremely unequal electron energy sharing [25-27]. The former results were fitted well using a heliumlike theory [28]; the latter results agreed reasonably well with $a b$ initio calculations that coherently superposed amplitudes for orthogonal linear polarizations [29].

In this Letter, we provide an analytic formulation for DPI of $\mathrm{H}_{2}$ in its ${ }^{1} \Sigma_{g}^{+}$ground state (binding energy $E_{g} \simeq 51.4 \mathrm{eV}$ and nuclear separation $R \simeq 1.4$ a.u.) by an elliptically polarized extreme ultraviolet pulse. We show that the elliptic dichroism [i.e., the difference of the sixfold differential probabilities (SDPs) for opposite pulse helicities] is comprised of two terms: an atomiclike dichroism (AD) term and a molecular symmetry-mixed dichroism (MSMD) term. The AD effect, involving only the ${ }^{1} \Pi_{u}^{+}$ ionization amplitudes, has the same form as for DPI of $\mathrm{He}$ $[3,5,12,13]$. In contrast, the MSMD effect originates from an interplay of the ${ }^{1} \Sigma_{u}^{+}$and ${ }^{1} \Pi_{u}^{+}$ionization amplitudes, which may be comparable at the low photon energy of $75 \mathrm{eV}$ considered here. Except when the light propagation direction $\hat{\mathbf{k}}$ is along the molecular axis $\mathbf{R}$, the MSMD effect is predicted to be nonzero. Moreover, we identify electron detection geometries in which the $\mathrm{AD}$ vanishes, such as in Figs. 1(a) and 1(b), thus allowing the MSMD to be studied directly. For those geometries, the MSMD occurs in both

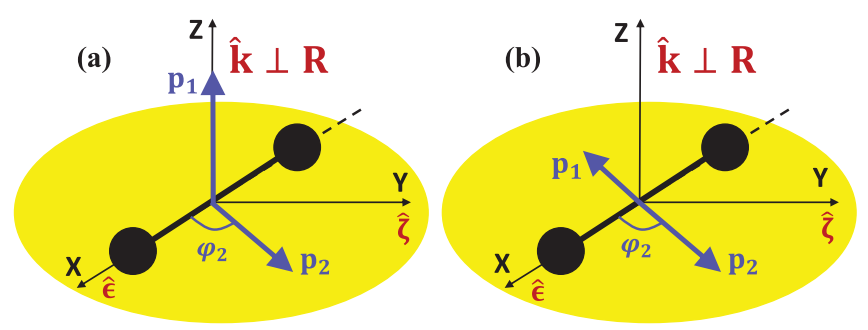

FIG. 1. Two geometries for detecting the outgoing electron momenta, $\mathbf{p}_{1}$ and $\mathbf{p}_{2}$, that allow direct access, measurement, and control of the MSMD in DPI of fixed-in-space $\mathrm{H}_{2}$ by an elliptically polarized light pulse whose propagation direction, $\hat{\mathbf{k}} \| \hat{\mathbf{z}}$, is perpendicular to the molecular vector, $\mathbf{R} \| \hat{\mathbf{x}}$. (a) Perpendicular electron ejection with $\mathbf{p}_{1} \| \hat{\mathbf{k}}$ and $\mathbf{p}_{2}$ in the laser polarization plane $(\hat{\boldsymbol{\epsilon}}, \hat{\boldsymbol{\zeta}})$ with spherical angles, $\theta_{2}=\pi / 2, \varphi_{2}$. (b) Back-to-back electron ejection in the polarization plane with $\theta_{1}=\theta_{2}=\pi / 2$ and $\varphi_{2}=\varphi_{1}-\pi$. In all panels, $0 \leq \varphi_{2} \leq 2 \pi$ and we fix $\hat{\mathbf{k}}\|\hat{\mathbf{z}}, \hat{\boldsymbol{\epsilon}}\| \hat{\mathbf{x}}$, and $\hat{\boldsymbol{\zeta}} \| \hat{\mathbf{y}}$. The origin of coordinates is at the center of mass of the nuclei. 
the electron momentum and angular distributions for any energy sharing and can be controlled by tuning the pulse ellipticity. These predictions are illustrated by $a b$ initio fixed-nuclei numerical calculations of the full-dimensional time-dependent Schrödinger equation (TDSE), as described in [30]. We note that the detection geometries used in prior studies of the DPI angular distributions for $\mathrm{H}_{2}$ [23-29] involve contributions from both $\mathrm{AD}$ and MSMD terms.

For the pulse parameters used here, a perturbation theory (PT) analysis is valid. For one-photon DPI of $\mathrm{H}_{2}$ by a laser pulse with peak intensity below $10^{14} \mathrm{~W} / \mathrm{cm}^{2}$ having rightor left-circular polarization (RCP or LCP) and propagating along the molecular axis $\hat{\mathbf{k}} \| \mathbf{R}$, only transitions ${ }^{1} \Sigma_{g}^{+} \rightarrow{ }^{1} \Pi_{u}^{+}$ are allowed by electric-dipole selection rules, with $\Delta M=+1$ for RCP or $\Delta M=-1$ for LCP. For elliptically polarized light with $\hat{\mathbf{k}}$ not along $\mathbf{R}$ (e.g., $\hat{\mathbf{k}} \perp \mathbf{R}$ ), $\Delta M=0, \pm 1$ transitions ${ }^{1} \Sigma_{g}^{+} \rightarrow\left({ }^{1} \Sigma_{u}^{+},{ }^{1} \Pi_{u}^{+}\right)$are allowed. The first-order PT DPI amplitude $\mathcal{A}$ for an elliptically polarized pulse can be parametrized in terms of the vectors of the problem: i.e., the directions of the electron momenta, $\hat{\mathbf{p}}_{1}, \hat{\mathbf{p}}_{2}$, the internuclear axis at the instant of photoionization (and hence the proton recoil axis), $\hat{\mathbf{R}}$, and the polarization vector, $\mathbf{e}$, where $\mathbf{e}=(\hat{\boldsymbol{\epsilon}}+i \eta \hat{\zeta}) / \sqrt{1+\eta^{2}}$. Here $\hat{\boldsymbol{\epsilon}}$ and $\hat{\boldsymbol{\zeta}}=[\hat{\mathbf{k}} \times \hat{\boldsymbol{\epsilon}}]$ are the major and minor axes of the polarization ellipse, and $\eta$ is the pulse ellipticity: $\eta=+1$ for a RCP pulse and $\eta=-1$ for a LCP pulse. The result for $\mathcal{A}$ is $[28,31]$

$$
\begin{aligned}
\mathcal{A}= & e^{-i \phi}\left\{\left[a_{1}(\chi)\left(\hat{\mathbf{p}}_{1} \cdot \mathbf{e}\right)+a_{2}(\chi)\left(\hat{\mathbf{p}}_{2} \cdot \mathbf{e}\right)\right]\right. \\
& \left.+(\hat{\mathbf{R}} \cdot \mathbf{e})\left[b_{1}(\chi)\left(\hat{\mathbf{R}} \cdot \hat{\mathbf{p}}_{1}\right)+b_{2}(\chi)\left(\hat{\mathbf{R}} \cdot \hat{\mathbf{p}}_{2}\right)\right]\right\} .
\end{aligned}
$$

The coefficients $a_{j}, b_{j}$ are related to the notation of Ref. [28] as follows: $a_{j} \equiv A_{\Pi, j}, b_{j} \equiv A_{\Sigma, j}-A_{\Pi, j}$, and $j=1,2$. The dynamical amplitudes $A_{\Pi, j}$ and $A_{\Sigma, j}$ are true scalar functions of $\chi \equiv\left(p_{1}, p_{2}, R, u, u_{1}, u_{2}\right)$, where $u \equiv\left(\hat{\mathbf{p}}_{1} \cdot \hat{\mathbf{p}}_{2}\right), u_{1,2} \equiv\left(\hat{\mathbf{R}} \cdot \hat{\mathbf{p}}_{1,2}\right)$, and $p_{1,2}$ are the magnitudes of the electron momenta. The amplitudes $A_{\Pi, j}$ and $A_{\Sigma, j}$ depend upon the pulse parameters except for the carrier-envelope phase (CEP), $\phi$.

The amplitude (1) is valid for any orientation of the vectors $\hat{\mathbf{p}}_{1}, \hat{\mathbf{p}}_{2}$, and $\hat{\mathbf{R}}$. Using Eq. (1), a general parametrization of the SDP, $\mathcal{W}(\mathbf{e})=|\mathcal{A}|^{2}$, has the form

$$
\begin{aligned}
\mathcal{W}(\mathbf{e})= & \left|a_{1}\right|^{2}\left|\hat{\mathbf{p}}_{1} \cdot \mathbf{e}\right|^{2}+\left|a_{2}\right|^{2}\left|\hat{\mathbf{p}}_{2} \cdot \mathbf{e}\right|^{2}+|\hat{\mathbf{R}} \cdot \mathbf{e}|^{2}|b|^{2} \\
& +2 \operatorname{Re}\left[\left(a_{1} a_{2}^{*}\right)\left(\hat{\mathbf{p}}_{1} \cdot \mathbf{e}\right)\left(\hat{\mathbf{p}}_{2} \cdot \mathbf{e}^{*}\right)\right] \\
& +2 \operatorname{Re}\left[\left(a_{1} b^{*}\right)\left(\hat{\mathbf{p}}_{1} \cdot \mathbf{e}\right)\left(\hat{\mathbf{R}} \cdot \mathbf{e}^{*}\right)\right] \\
& +2 \operatorname{Re}\left[\left(a_{2} b^{*}\right)\left(\hat{\mathbf{p}}_{2} \cdot \mathbf{e}\right)\left(\hat{\mathbf{R}} \cdot \mathbf{e}^{*}\right)\right]
\end{aligned}
$$

where $b=b_{1}\left(\hat{\mathbf{R}} \cdot \hat{\mathbf{p}}_{1}\right)+b_{2}\left(\hat{\mathbf{R}} \cdot \hat{\mathbf{p}}_{2}\right)$. Taking the difference of the SDP (2) for $\mathbf{e}$ and $\mathbf{e}^{*}, \Delta \mathcal{W}=\mathcal{W}(\mathbf{e})-\mathcal{W}\left(\mathbf{e}^{*}\right)$, gives an expression for the absolute elliptic dichroism,

$$
\begin{aligned}
\Delta \mathcal{W}= & 4 \operatorname{Im}\left[a_{1}^{*} a_{2}\right] \operatorname{Im}\left[\left(\hat{\mathbf{p}}_{1} \cdot \mathbf{e}\right)\left(\hat{\mathbf{p}}_{2} \cdot \mathbf{e}^{*}\right)\right] \\
& +4 \operatorname{Im}\left[a_{1}^{*} b\right] \operatorname{Im}\left[\left(\hat{\mathbf{p}}_{1} \cdot \mathbf{e}\right)\left(\hat{\mathbf{R}} \cdot \mathbf{e}^{*}\right)\right] \\
& +4 \operatorname{Im}\left[a_{2}^{*} b\right] \operatorname{Im}\left[\left(\hat{\mathbf{p}}_{2} \cdot \mathbf{e}\right)\left(\hat{\mathbf{R}} \cdot \mathbf{e}^{*}\right)\right]
\end{aligned}
$$

From the definition of $\mathbf{e}$ above, the factors on the right of each term in (3) may be expressed in terms of the degree of circular polarization, $\xi \equiv i \hat{\mathbf{k}} \cdot\left[\mathbf{e} \times \mathbf{e}^{*}\right]=2 \eta /\left(1+\eta^{2}\right)$,

$$
\operatorname{Im}\left[\left(\hat{\mathbf{q}}_{1} \cdot \mathbf{e}\right)\left(\hat{\mathbf{q}}_{2} \cdot \mathbf{e}^{*}\right)\right]=\frac{\xi}{2}\left(\hat{\mathbf{k}} \cdot\left[\hat{\mathbf{q}}_{1} \times \hat{\mathbf{q}}_{2}\right]\right) .
$$

Using (4), the elliptic dichroism (3) can be expressed as

$$
\Delta \mathcal{W}=\Delta \mathcal{W}_{\mathrm{AD}}+\Delta \mathcal{W}_{\mathrm{MSMD}}
$$

where $\Delta \mathcal{W}_{\mathrm{AD}}$ is the atomiclike contribution $[3,5,12,13]$,

$$
\Delta \mathcal{W}_{\mathrm{AD}}=2 \operatorname{Im}\left[A_{\Pi, 1}^{*}(\chi) A_{\Pi, 2}(\chi)\right] \xi \hat{\mathbf{k}} \cdot\left[\hat{\mathbf{p}}_{1} \times \hat{\mathbf{p}}_{2}\right],
$$

and where $\Delta \mathcal{W}_{\mathrm{MSMD}}$ is unique to the molecule,

$$
\begin{aligned}
\Delta \mathcal{W}_{\mathrm{MSMD}} & =\xi \hat{\mathbf{k}} \cdot\left\{c_{1}\left[\hat{\mathbf{p}}_{1} \times \hat{\mathbf{R}}\right]+c_{2}\left[\hat{\mathbf{p}}_{2} \times \hat{\mathbf{R}}\right]\right\} \\
& =\xi[\hat{\mathbf{R}} \times \hat{\mathbf{k}}] \cdot\left\{c_{1} \hat{\mathbf{p}}_{1}+c_{2} \hat{\mathbf{p}}_{2}\right\},
\end{aligned}
$$

with the coefficients $c_{j}(j=1,2)$ given by

$$
\begin{aligned}
c_{j}= & 2 \operatorname{Im}\left[A _ { \Pi , j } ^ { * } ( \chi ) \left\{\left[A_{\Sigma, 1}(\chi)-A_{\Pi, 1}(\chi)\right]\left(\hat{\mathbf{R}} \cdot \hat{\mathbf{p}}_{1}\right)\right.\right. \\
& \left.\left.+\left[A_{\Sigma, 2}(\chi)-A_{\Pi, 2}(\chi)\right]\left(\hat{\mathbf{R}} \cdot \hat{\mathbf{p}}_{2}\right)\right\}\right] .
\end{aligned}
$$

Both the AD (6) and MSMD (7) terms are proportional to $\xi \hat{\mathbf{k}}$, the time-odd pseudovector of the incident light pulse that is responsible for dichroic effects [12]. The vector structures of the AD and MSMD terms ensure that both $\Delta \mathcal{W}_{\mathrm{AD}}$ and $\Delta \mathcal{W}_{\mathrm{MSMD}}$ are time-even true scalars. Also, Eqs. (6)-(9) demonstrate that the AD effect stems from interference of the atomiclike ${ }^{1} \Pi_{u}^{+}$continuum amplitudes, while the MSMD effect involves interference of the molecular ${ }^{1} \Sigma_{u}^{+}$and ${ }^{1} \Pi_{u}^{+}$continuum amplitudes.

Analysis of Eqs. (6)-(9) gives selection rules specifying when the $\mathrm{AD}$ and/or MSMD effects vanish. The $\mathrm{AD}$ (6) vanishes when (i) $\operatorname{Im}\left[A_{\Pi, 1}^{*}(\chi) A_{\Pi, 2}(\chi)\right]=0$, which is the case for equal energy sharing (EES), since $A_{\Pi, 1}=A_{\Pi, 2}$ for $p_{1}=p_{2}$, and is also the case for the approximate representation of the outgoing electrons by plane waves, since then both $A_{\Pi, 1}$ and $A_{\Pi, 2}$ are real [13], and (ii) $\hat{\mathbf{k}} \cdot\left[\hat{\mathbf{p}}_{1} \times \hat{\mathbf{p}}_{2}\right]=0$, which is the case for detection of one of the electrons along $\hat{\mathbf{k}}$ [e.g., $\hat{\mathbf{p}}_{1} \| \hat{\mathbf{k}}$ in Fig. 1(a)], for $\hat{\mathbf{k}}$ lying in the detection plane, and for collinear detection of the electron momenta [e.g., $\hat{\mathbf{p}}_{1}=-\hat{\mathbf{p}}_{2}$ in Fig. 1(b)]. The MSMD (8) vanishes when (iii) $[\hat{\mathbf{R}} \times \hat{\mathbf{k}}]=0$, which is the case for $\hat{\mathbf{k}} \| \hat{\mathbf{R}}$, (iv) $[\hat{\mathbf{R}} \times \hat{\mathbf{k}}] \cdot \hat{\mathbf{p}}_{1,2}=0$, which is the case when both the molecular axis $\hat{\mathbf{R}}$ and the beam direction $\hat{\mathbf{k}}$ 
lie in the detection plane, and (v) $c_{1}=c_{2}=0$, which occurs when the molecular axis is perpendicular to the detection plane $\left(\hat{\mathbf{R}} \cdot \hat{\mathbf{p}}_{1,2}=0\right)$. Note that $\Delta \mathcal{W}$ (5) vanishes for the case of EES and detecting the electrons in the plane perpendicular to the molecular axis, which fact can thus serve as a probe of molecular orientation. Averaging $\Delta \mathcal{W}(5)$ over the molecular orientation reduces its vector structure to the same form as for the AD effect $[21,33]$.

To study the MSMD, we choose detection geometries in which $\hat{\mathbf{k}} \perp \mathbf{R}$ [such as in Figs. 1(a) and 1(b)] so that the $\mathrm{AD}$ (6) vanishes according to selection rule (ii). For those geometries, using Eqs. (8) and (9) the MSMD becomes

$$
\Delta \mathcal{W}_{\mathrm{MSMD}}^{\perp}=\xi \operatorname{Im}\left[A_{\Pi}^{*}(\chi) A_{\Sigma}(\chi)\right] \sin \left(2 \varphi_{2}\right) \sin ^{2} \theta_{2},
$$

where $A_{\Sigma}(\chi) \equiv A_{\Sigma, 2}(\chi)$ and $A_{\Pi}(\chi) \equiv A_{\Pi, 2}(\chi)$ for the geometry in Fig. 1(a), and $A_{\Sigma}(\chi) \equiv A_{\Sigma, 2}(\chi)-A_{\Sigma, 1}(\chi)$ and $A_{\Pi}(\chi) \equiv A_{\Pi, 2}(\chi)-A_{\Pi, 1}(\chi)$ for the geometry in Fig. 1(b). For these two geometries, the $\operatorname{SDP} \mathcal{W}^{\perp}(\mathbf{e})$ in Eq. (2) is

$$
\begin{aligned}
\mathcal{W}^{\perp}(\mathbf{e})= & {[(1+\ell) / 2]\left|A_{\Sigma}(\chi)\right|^{2} \cos ^{2} \varphi_{2} \sin ^{2} \theta_{2} } \\
& +[(1-\ell) / 2]\left|A_{\Pi}(\chi)\right|^{2} \sin ^{2} \varphi_{2} \sin ^{2} \theta_{2} \\
& +(1 / 2) \Delta \mathcal{W}{ }_{\mathrm{MSMD}}^{\perp},
\end{aligned}
$$

where $\ell \equiv(\mathbf{e} \cdot \mathbf{e})=\left(1-\eta^{2}\right) /\left(1+\eta^{2}\right)$ is the linear polarization degree. [Note that RCP (LCP) corresponds to $\xi=$ $+1(-1)$ and $\ell=0$, while linear polarization corresponds to $\xi=0$ and $\ell=1$.] Observe that the first two terms in (11) are invariant to $\xi \rightarrow-\xi$ (or $\eta \rightarrow-\eta$ ) while the third term $\left[\propto \Delta \mathcal{W}_{\mathrm{MSMD}}^{\perp}(10)\right]$ changes sign. Thus, it is the third term that is responsible for the MSMD effect in the momentum distribution (11), as demonstrated numerically by our TDSE results in Fig. 2 for the geometry in Fig. 1(a), in which $\mathbf{p}_{1} \perp \mathbf{p}_{2}$ and $\mathbf{p}_{1}\|\hat{\mathbf{k}}\| \hat{\mathbf{z}}$.

Our numerical SDP results for a pulse with elliptical polarization e are obtained by the projection [30,31],

$\mathcal{W}^{\perp}(\mathbf{e})=\left|\left\langle\Phi_{\mathbf{p}_{1}, \mathbf{p}_{2}}^{(-)}\left(\mathbf{r}_{1}, \mathbf{r}_{2}\right) \mid \Psi_{C}\left(\mathbf{r}_{1}, \mathbf{r}_{2} ; R, T+T_{p}\right)\right\rangle\right|^{2}$.

Here $\mathbf{r}_{1}$ and $\mathbf{r}_{2}$ are the electron coordinates for an origin at the center of mass of the nuclei; $\Psi_{C}\left(\mathbf{r}_{1}, \mathbf{r}_{2} ; R, T+T_{p}\right)$ is the doubly ionized part of the wave packet $\Psi\left(\mathbf{r}_{1}, \mathbf{r}_{2} ; R, t\right)$ solution of the TDSE at a time $T_{p} \geq 25$ a.u. after the end of a pulse with duration $T \simeq 331$ as; and $\Phi_{\mathbf{p}_{1}, \mathbf{p}_{2}}^{(-)}$is the field-free double-continuum final state with excess energy $E$ approximated by a product of two Coulomb waves with charge $Z=2 \quad[34,35]$. The six-cycle cosine-squared pulse has carrier frequency $\omega=75 \mathrm{eV}$, peak intensity $I=50 \mathrm{TW} / \mathrm{cm}^{2}$, and CEP $\phi=0$. Results for the geometry in Fig. 1(b) are given elsewhere.

Our TDSE results (12) for the $\mathbf{p}_{2}$ distributions in the laser polarization $x-y$ plane produced by RCP and LCP attosecond pulses are shown respectively in Figs. 2(a) and 2(c), and Figs. 2(b) and 2(d). Owing to the broad bandwidth $\Delta \omega \simeq 18 \mathrm{eV}$ of the attosecond pulses, the two photoelectrons share the excess energy $0.1 \leq E \leq 41.6 \mathrm{eV}$. Figures 2(a) and 2(b) show results for unequal energy
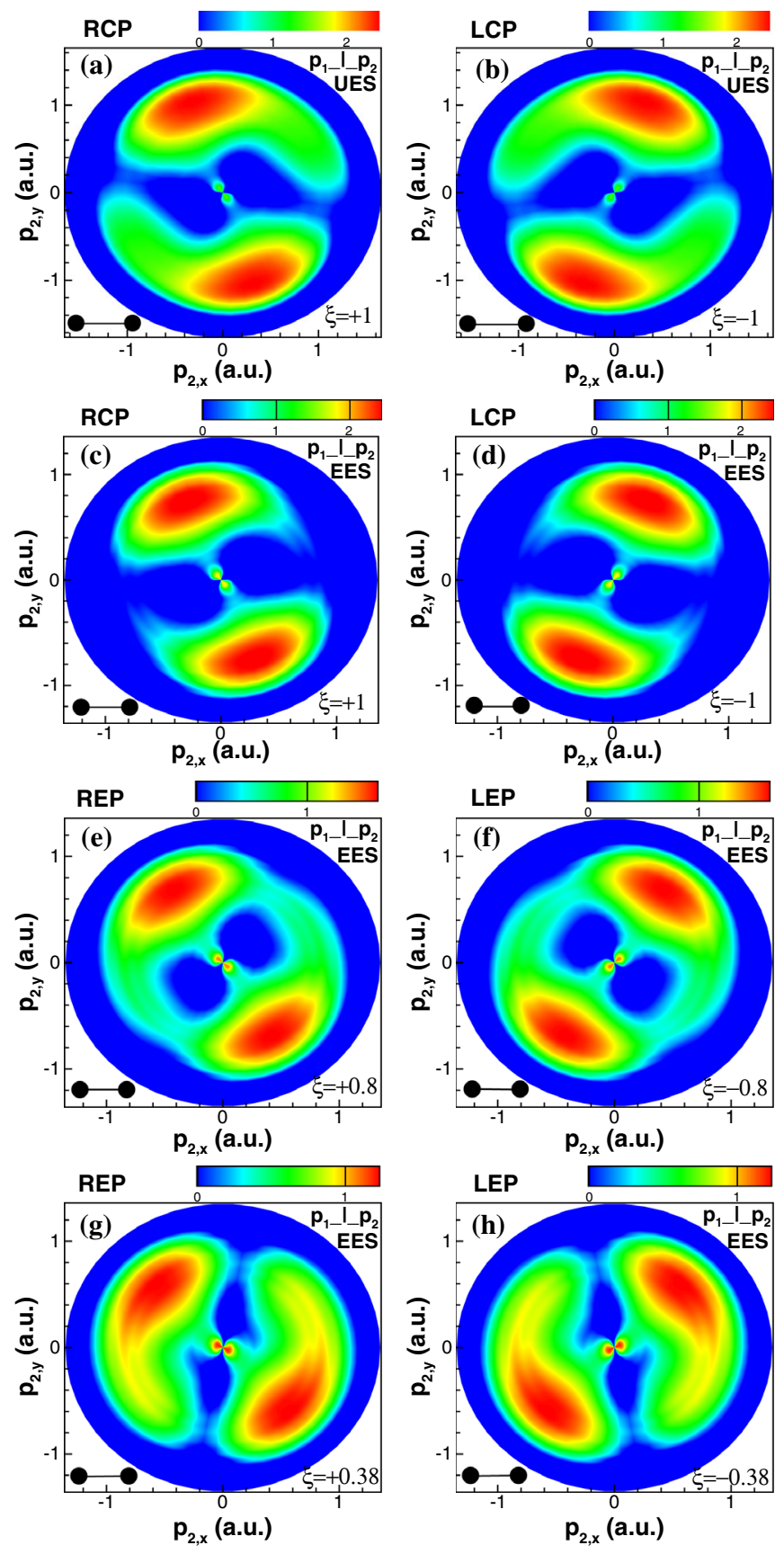

FIG. 2. TDSE results (12) (in units of $10^{-8}$ a.u.) for the $\mathbf{p}_{2}$ distributions in the polarization $x y$ plane for DPI of $\mathrm{H}_{2}$ by a right(column 1) or left-handed (column 2) elliptically polarized (REP or LEP) attosecond pulse, with $\hat{\mathbf{k}} \perp \mathbf{R}$ (indicated by the dumbbell shape), for the geometry in Fig. 1(a). The circular polarization degree is $\xi= \pm 1$ (rows 1,2 ), $\xi= \pm 0.8$ (row 3 ), and $\xi= \pm 0.38$ (row 4). The two electrons share the excess energy $0.1 \leq E \leq$ $41.6 \mathrm{eV}$ in the ratio $\varepsilon \equiv E_{1} / E$ : row 1 (UES), $\varepsilon=25 \%$; rows $2,3,4$ (EES), $\varepsilon=50 \%$. 
sharing (UES) fixed at $\varepsilon \equiv E_{1} / E=25 \%$, whereas Figs. 2(c) and 2(d) show results for EES, i.e., $\varepsilon=50 \%$. For each energy sharing, the $\mathbf{p}_{2}$ distributions produced by the RCP [Figs. 2(a) and 2(c)] and LCP [Figs. 2(b) and 2(d)] pulses are mirror images of one another, due to the MSMD effect. One observes that each of the $\mathbf{p}_{2}$ distributions in Figs. 2(a)2(d) exhibits a distorted dipolelike pattern tilted at an angle to the molecular $x$ axis, with the UES case showing the greatest distortion. These features of our TDSE results may be interpreted using PT formulas (10) and (11) as resulting from an interplay between the ionization amplitudes $A_{\Sigma}(\chi)$ and $A_{\Pi}(\chi)$, where $\chi=\left(p_{1}, p_{2} ; R ; 0,0, \cos \varphi_{2}\right)$, which have comparable magnitudes [30] but enter (11) with different angular dependences. For both EES and UES, the ${ }^{1} \Pi_{u}^{+}$ contribution [second term in (11)] has a dipolelike shape along the $y$ axis (see Fig. 4 in Ref. [30]). The ${ }^{1} \Sigma_{u}^{+}$ contribution [first term in (11)] exhibits a four-lobe shape along the molecular $x$ axis for EES (as found in [34-36]) and a six-lobe shape for UES (as shown by Fig. 4 in Ref. [30]). Moreover, the ratio (about 2) of the magnitudes of the ${ }^{1} \Pi_{u}^{+}$and ${ }^{1} \Sigma_{u}^{+}$amplitudes is a bit higher for EES than for UES. Thus, for $\xi= \pm 1$ the smaller contribution of the ${ }^{1} \Sigma_{u}^{+}$amplitude is less visible for EES than for UES. For both UES and EES cases, the MSMD term (10), $\propto \xi\left|A_{\Pi}(\chi) A_{\Sigma}(\chi)\right| \sin \psi$, where $\psi$ is the relative phase of the amplitudes, induces a counterclockwise (for $\xi=+1$ ) or clockwise (for $\xi=-1$ ) rotation relative to the $y$ axis.

The relative contributions of the $A_{\Sigma}$ and $A_{\Pi}$ amplitudes in (11) may be controlled by varying the pulse ellipticity $\eta$. For circular polarization, $\ell=0$, so that the first and second terms in (11) have the same coefficient, $1 / 2$. As $\eta$ decreases from unity, $\ell$ increases, thus increasing the $A_{\Sigma}$ contribution in the first line of (11) and decreasing the $A_{\Pi}$ contribution in the second line. Our TDSE results for EES in Figs. 2(e) and 2(f) for $\xi= \pm 0.8(\eta= \pm 0.5)$ and in Figs. 2(g) and 2(h) for $\xi= \pm 0.38(\eta= \pm 0.2)$ show the dramatic change in shape of the $\mathbf{p}_{2}$ distributions as $\eta$ varies. The decrease of $\xi$ from unity to 0.38 changes the distribution from the dipolelike shape in Figs. 2(c) and 2(d) to the yin-yanglike pattern in Figs. 2(g) and 2(h).

The MSMD effect is also clearly seen in the energyintegrated angular distributions obtained from the $\mathbf{p}_{2}$ distributions in Figs. 2(a) and 2(b) or Figs. 2(c) and 2(d), as shown for opposite helicities in Fig. 3(a) for UES and Fig. 3(b) for EES. Their difference (i.e., the energy-integrated MSMD) is shown in Fig. 3(c) for both EES $(\varepsilon=0.5)$ and UES $(\varepsilon=0.25)$. The energy-integrated MSMD curves in Fig. 3(c) exhibit inversion symmetry; hence, they vanish if integrated over $\varphi_{2}$, as is characteristic of dichroic phenomena from achiral targets. One sees also that the angular structure of the energy-integrated MSMD depends upon the energy sharing. For both EES and UES, there are five common geometrical 0's at $\varphi_{2}=0, \pi / 2,3 \pi / 2, \pi, 2 \pi$, originating from 0 's of the $\sin \left(2 \varphi_{2}\right)$ factor in $\Delta \mathcal{W}_{\text {MSMD }}^{\perp}$ (10). While dynamical 0 's
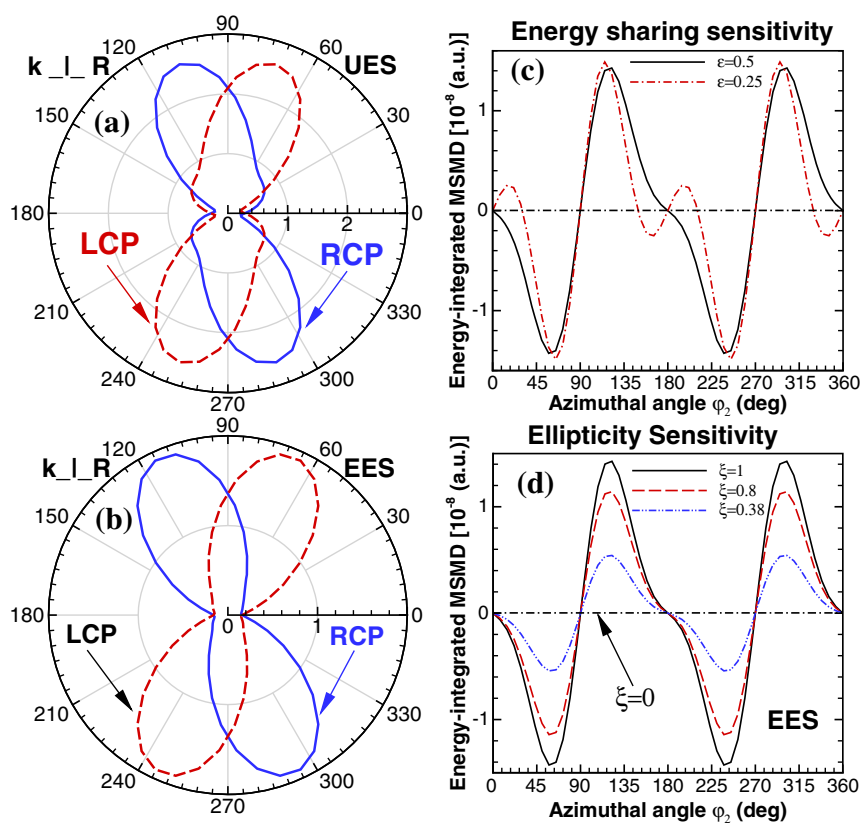

FIG. 3. (a) Energy-integrated angular distributions for RCP (solid line) and LCP (dashed line) obtained from the SDPs shown in Figs. 2(a) and 2(b) for UES; (b) Corresponding results for EES from Figs. 2(c) and 2(d); (c) Energy-sharing sensitivity of the energy-integrated MSMD [obtained as the RCP-LCP difference of the results in each of (a) and (b)]; (d) Dependence of the energy-integrated MSMD for EES on the degree of circular polarization $\xi$.

are absent for EES in Fig. 3(c), four dynamical 0's at $\varphi_{2} \simeq 33^{\circ}, 147^{\circ}, 213^{\circ}, 327^{\circ}$ are found for UES, which stem from 0 's of the dynamical factor $\operatorname{Im}\left[A_{\Pi}^{*}(\chi) A_{\Sigma}(\chi)\right]$ in $\Delta \mathcal{W}_{\mathrm{MSMD}}^{\perp}$ (10). Dynamical 0's and their sensitivity to excess energy and energy sharing have been predicted $[4,11,13]$ and measured [11] for CD in He by monochromatic circularly polarized fields.

The $\varphi_{2}$-dependence of the energy-integrated MSMD for the three values of $|\xi|$ used in Fig. 2 for the case of EES is shown in Fig. 3(d). In agreement with the PT formula (10), Fig. 3(d) shows that the energy-integrated MSMD scales linearly with $\xi$. (This result holds also for any UES case.) Also, TDSE results (not shown) confirm that the MSMD scales linearly with the peak pulse intensity $I$, in agreement with the PT formula (10) since each amplitude in the factor $\operatorname{Im}\left[A_{\Pi}^{*}(\chi) A_{\Sigma}(\chi)\right]$ scales as $\sqrt{I}$. To quantify the MSMD, the normalized energy-integrated MSMD can be evaluated. The MSMD is large for any energy sharing, but is larger for EES than for UES, reaching a maximum of $70 \%$ for the EES case in Fig. 3(d).

In summary, we have formulated the dichroism in singlephoton DPI of $\mathrm{H}_{2}$ by an elliptically polarized ultrashort pulse as a sum of AD and MSMD terms. For detection geometries in which the $\mathrm{AD}$ term vanishes, we have predicted analytically, and numerically confirmed, that the MSMD effect is significant in both the momentum 
and angular distributions and controllable by the pulse ellipticity. The MSMD effect originates from an interference of the $A_{\Pi}$ and $A_{\Sigma}$ amplitudes and depends linearly on the pulse degree of circular polarization, $\xi$, on the pulse peak intensity, and on $\sin \psi$, where $\psi$ is the relative phase of the two amplitudes. It occurs for any electron energysharing configuration and for all incident laser beam propagation directions $\hat{\mathbf{k}}$ except for $\hat{\mathbf{k}} \| \mathbf{R}$. Although attosecond pulses were used in our calculations (and isolated attosecond pulses with controlled polarization have been realized [37]), our PT analysis applies for any pulse duration. Note that vibrational or rotational motions occur typically on timescales respectively of $10^{-13} \mathrm{~s}$ or $10^{-10} \mathrm{~s}$ [38] so that the molecular symmetries would be unchanged for pulses of a few fs, although the vibrational motion may affect the MSMD magnitude. Longer pulse durations, allowing for rotational motion, would necessitate averaging the PT results over molecular orientation, as noted above. Experiments for DPI of aligned $\mathrm{H}_{2}$ molecules by circularly polarized light [25-27], which have noted evidence of CD in the electron angular distributions, can confirm our predictions for $\mathrm{AD}$ and MSMD effects by using the detection geometries specified in this work.

Research of A.F.S. and J.M. N. D. is supported in part by the U.S. Department of Energy (DOE), Office of Science, Basic Energy Sciences (BES), under Award No. DE-FG03-96ER14646; research of A. V. M. and N. L.M. is supported by the Russian Ministry of Education and Science under Grants No. 3.1761.2017/ 4.6 (A. V. M.) and No. 3.7514.2017/8.9 (N. L. M.); research of S.X.H. is supported by the DOE National Nuclear Security Administration under Award No. DENA0003856, the University of Rochester, and the New York State Energy Research and Development Authority; research of L. B. M. is supported by the VKR Center of Excellence, QUSCOPE; computations were done using Crane of the Holland Computing Center at the University of Nebraska-Lincoln, and Stampede 2 at TACC under Grant No. PHY-120003.

* Corresponding author.

marcelngoko@unl.edu

${ }^{\dagger}$ Deceased.

[1] J. S. Briggs and V. Schmidt, Differential cross sections for photo-double-ionization of the helium atom, J. Phys. B 33, R1 (2000).

[2] J. Berakdar and H. Klar, Chiral multi-electron emission, Phys. Rep. 340, 473 (2001).

[3] J. Berakdar and H. Klar, Circular Dichroism in Double Photoionization, Phys. Rev. Lett. 69, 1175 (1992).

[4] J. Berakdar, H. Klar, A. Huetz, and P. Selles, Chiral electron pairs from double photoionization, J. Phys. B 26, 1463 (1993).
[5] N. L. Manakov, S. I. Marmo, and A. V. Meremianin, A new technique in the theory of angular distributions in atomic processes: the angular distribution of photoelectrons in single and double photoionization, J. Phys. B 29, 2711 (1996).

[6] J. Viefhaus et al., Experimental Evidence for Circular Dichroism in the Double Photoionization of Helium, Phys. Rev. Lett. 77, 3975 (1996).

[7] V. Mergel et al., Helicity Dependence of the PhotonInduced Three-Body Coulomb Fragmentation of Helium Investigated by Cold Target Recoil Ion Momentum Spectroscopy, Phys. Rev. Lett. 80, 5301 (1998).

[8] A. S. Kheifets and I. Bray, Calculation of Circular Dichroism in Helium Double Photoionization, Phys. Rev. Lett. 81, 4588 (1998).

[9] K. Soejima, A. Danjo, K. Okuno, and A. Yagishita, Linear and Circular Dichroism in the Double Photoionization of Helium, Phys. Rev. Lett. 83, 1546 (1999).

[10] A. Kheifets, I. Bray, K. Soejima, A. Danjo, K. Okuno, and A. Yagashita, Experimental and theoretical study of linear and circular dichroism in helium double photoionization, J. Phys. B 32, L501 (1999).

[11] M. Achler, V. Mergel, L. Spielberg, R. Dörner, Y. Azuma, and H. Schmidt-Böcking, Photo double ionization of He by circular and linear polarized single-photon absorption, J. Phys. B 34, 965 (2001).

[12] N. L. Manakov, M. V. Frolov, B. Borca, and A. F. Starace, Multiphoton detachment of a negative ion by an elliptically polarized, monochromatic laser field, J. Phys. B 36, R49 (2003).

[13] A. Y. Istomin, N. L. Manakov, and A. F. Starace, Perturbative analysis of the triply differential cross section and circular dichroism in photo-double-ionization of He, Phys. Rev. A 69, 032713 (2004).

[14] N. A. Cherepkov, Spin polarization of atomic and molecular photoelectrons, Adv. At. Mol. Phys. 19, 395 (1983).

[15] G. Schönhense and J. Hormes, Photoionization of oriented systems and circular dichroism, in $V U V$ and Soft-X-ray Photoionization, edited by U. Becker and D. A. Shirley (Plenum, New York, 1996), Chap. 17, pp. 607-652.

[16] N. Böwering, T. Lischke, B. Schmidtke, N. Müller, T. Khalil, and U. Heinzmann, Asymmetry in Photoelectron Emission from Chiral Molecules Induced by Circularly Polarized Light, Phys. Rev. Lett. 86, 1187 (2001).

[17] N. J. Greenfield, Using circular dichroism spectra to estimate protein secondary structure, Nat. Protoc. 1, 2876 (2006).

[18] G. A. Garcia, L. Nahon, S. Daly, and I. Powis, Vibrationally induced inversion of photoelectron forward-backward asymmetry in chiral molecule photoionization by circularly polarized light, Nat. Commun. 4, 2132 (2013).

[19] M. Wietstruk, A. Melnikov, C. Stamm, T. Kachel, N. Pontius, M. Sultan, C. Gahl, M. Weinelt, H. A. Dürr, and U. Bovensiepen, Hot-Electron-Driven Enhancement of Spin-Lattice Coupling in $\mathrm{Gd}$ and $\mathrm{Tb} 4 \mathrm{f}$ Ferromagnets Observed by Femtosecond X-Ray Magnetic Circular Dichroism, Phys. Rev. Lett. 106, 127401 (2011).

[20] C. von Korff Schmising et al., Imaging Ultrafast Demagnetization Dynamics after a Spatially Localized Optical Excitation, Phys. Rev. Lett. 112, 217203 (2014). 
[21] T. J. Reddish and J. M. Feagin, Photo double ionization of molecular deuterium, J. Phys. B 32, 2473 (1999).

[22] M. Walter, A. V. Meremianin, and J. S. Briggs, Multiparticle photoionization by a single photon, J. Phys. B 36, 4561 (2003).

[23] T. J. Reddish, J. P. Wightman, M. A. MacDonald, and S. Cvejanović, Triple Differential Cross Section Measurements for Double Photoionization of $\mathrm{D}_{2}$, Phys. Rev. Lett. 79, 2438 (1997).

[24] J. P. Wightman, S. Cvejanović, and T. J. Reddish, $(\gamma, 2 e)$ cross section measurements of $\mathrm{D}_{2}$ and He, J. Phys. B 31, 1753 (1998).

[25] D. Akoury et al., The simplest double slit: Interference and entanglement in double photoionization of $\mathrm{H}_{2}$, Science 318, 949 (2007).

[26] K. Kreidi et al., Interference in the Collective Electron Momentum in Double Photoionization of $\mathrm{H}_{2}$, Phys. Rev. Lett. 100, 133005 (2008).

[27] M. S. Schöffler et al., Photo-double-ionization of $\mathrm{H}_{2}$ : Twocenter interference and its dependence on the internuclear distance, Phys. Rev. A 78, 013414 (2008).

[28] J. M. Feagin, A heliumlike description of molecular hydrogen photo-double ionization, J. Phys. B 31, L729 (1998).

[29] D. A. Horner, S. Miyabe, T. N. Rescigno, C. W. McCurdy, F. Morales, and F. Martín, Classical Two-Slit Interference Effects in Double Photoionization of Molecular Hydrogen at High Energies, Phys. Rev. Lett. 101, 183002 (2008).

[30] J. M. Ngoko Djiokap, A. V. Meremianin, N. L. Manakov, L. B. Madsen, S.X. Hu, and A. F. Starace, Dynamical electron vortices in attosecond double photoionization of $\mathrm{H}_{2}$, Phys. Rev. A 98, 063407 (2018).

[31] See Supplemental Material at http://link.aps.org/ supplemental/10.1103/PhysRevLett.123.143202 for (i) a derivation of the parametrization in Eq. (1) for the DPI amplitude $\mathcal{A}$, which includes Ref. [32]; and (ii) a description of numerical methods used to solve the two-electron TDSE for DPI of $\mathrm{H}_{2}$ by an elliptically polarized laser pulse.

[32] L. D. Landau and E. M. Lifshitz, Quantum Mechanics, 3rd ed. (Pergamon, Oxford, 1977).

[33] N. Chandra, Circular dichroism in the double photoionization of molecules, Chem. Phys. Lett. 237, 545 (1995).

[34] I. A. Ivanov and A. S. Kheifets, Time-dependent calculations of double photoionization of the aligned $\mathrm{H}_{2}$ molecule, Phys. Rev. A 85, 013406 (2012).

[35] X. Guan, K. Bartschat, and B. I. Schneider, Breakup of the aligned $\mathrm{H}_{2}$ molecule by xuv laser pulses: A time-dependent treatment in prolate spheroidal coordinates, Phys. Rev. A 83, 043403 (2011).

[36] J. M. Feagin, J. Colgan, A. Huetz, and T. J. Reddish, Electron-Pair Excitations and the Molecular Coulomb Continuum, Phys. Rev. Lett. 103, 033002 (2009).

[37] P.-C. Huang et al., Polarization control of isolated highharmonic pulses, Nat. Photonics 12, 349 (2018).

[38] M. Dantus, R. M. Bowman, and A. H. Zewail, Femtosecond laser observations of molecular vibration and rotation, Nature (London) 343, 737 (1990). 